\title{
Human Leukocyte Antigens and Biomarkers in Type 1 Diabetes Mellitus Induced by Immune-Checkpoint
}

\section{Inhibitors}

\author{
Hidefumi Inaba ${ }^{1,2}$, Yosuke Kaido ${ }^{1}$, Saya Ito ${ }^{1}$, Tomonao Hirobata ${ }^{1}$, Gen Inoue ${ }^{1}$, Takakazu Sugita ${ }^{3}$, Yuki Yamamoto ${ }^{4}$, \\ Masatoshi Jinnin $^{4}$, Hiroaki Kimura ${ }^{5}$, Tomoko Kobayashi ${ }^{6}$, Shintaro Iwama ${ }^{6}$, Hiroshi Arima ${ }^{6}$, Takaaki Matsuoka ${ }^{2}$ \\ ${ }^{1}$ Department of Diabetes and Endocrinology, Japanese Red Cross Wakayama Medical Center; ${ }^{2}$ The First Department of \\ Medicine, Wakayama Medical University; ${ }^{3}$ Department of Respiratory Medicine, Japanese Red Cross Wakayama Medical \\ Center; ${ }^{4}$ Department of Dermatology, Wakayama Medical University, Wakayama; ${ }^{5}$ Department of Pharmaceutical Health \\ Sciences, Kyushu University of Health and Welfare, Nobeoka; ${ }^{6}$ Department of Endocrinology and Diabetes, Nagoya University \\ Graduate School of Medicine, Nagoya, Japan
}

Background: Type 1 diabetes mellitus induced by immune-checkpoint inhibitors (ICI-T1DM) is a rare critical entity. However, the etiology of ICI-T1DM remains unclear.

Methods: In order to elucidate risk factors for ICI-T1DM, we evaluated the clinical course and immunological status of patients with ICI-T1DM who had been diagnosed during 2016 to 2021.

Results: Seven of 871 (0.8\%, six men and one woman) patients developed ICI-T1DM. We revealed that the allele frequencies of human leukocyte antigen (HLA)-DPA1*02:02 and DPB1*05:01 were significantly higher in the patients with ICI-T1DM In comparison to the controls who received ICI ( $11 / 14$ vs. $10 / 26, P=0.022 ; 11 / 14$ vs. $7 / 26, P=0.0027$, respectively). HLA-DRB $1 * 04: 05$, which has been found to be a T1DM susceptibility allele in Asians, was also observed as a high-risk allele for ICI-T1DM. The significance of the HLA-DPB1*05:01 and DRB1*04:05 alleles was confirmed by an analysis of four additional patients. The absolute/relative neutrophil count, neutrophils-lymphocyte ratio, and neutrophil-eosinophil ratio increased, and the absolute lymphocyte count and absolute/relative eosinophil count decreased at the onset as compared with 6 weeks before. In two patients, alterations in cytokines and chemokines were found at the onset.

Conclusion: Novel high-risk HLA alleles and haplotypes were identified in ICI-T1DM, and peripheral blood factors may be utilized as biomarkers.

Keywords: Immune checkpoint inhibitors; Immune-related adverse events; Diabetes mellitus, type 1; Biomarkers; Cytokines; Chemokines

Received: 1 October 2021, Revised: 22 November 2021, Accepted: 27 December 2021

Corresponding author: Hidefumi Inaba

The First Department of Medicine, Wakayama Medical University, 811-1, Kimiidera, Wakayama 641-8509, Japan

Tel: +81-73-441-0625, Fax: +81-73-445-9436

E-mail: inaba@wakayama-med.ac.jp

\section{Copyright $@ 2022$ Korean Endocrine Society}

This is an Open Access article distributed under the terms of the Creative Commons Attribution Non-Commercial License (https://creativecommons.org/ licenses/by-nc/4.0/) which permits unrestricted non-commercial use, distribution, and reproduction in any medium, provided the original work is properly cited. 


\section{INTRODUCTION}

Immune-checkpoint inhibitors (ICIs) are effective agents in cancer immunotherapy $[1,2]$. They augment anti-cancer immunity by inhibiting the binding of immune-checkpoint molecules, such as cytotoxic T-lymphocyte-associated antigen 4 (CTLA-4), programmed death protein 1 (PD-1), and ligand for PD-1 (PDL1) $[1,2]$. Immune-related adverse events (irAEs) often occur during treatment with ICIs; the incidence is more than $50 \%$ for any grade irAEs due to ICI treatment [3-8]. In endocrine organs, irAEs have been reported in the pituitary gland, the thyroid gland, the parathyroid glands, the adrenal glands, and the pancreas [3-8]. As biomarkers of irAEs, Fujisawa et al. [9] reported alterations in peripheral blood cell counts during irAEs. Regarding ICI-induced type 1 diabetes mellitus (ICI-T1DM), Takada et al. [10] found that female sex and malignant melanoma (MM) were risk factors for ICI-T1DM. We have reported biomarkers [7] and the predisposing role of human leukocyte antigen (HLA) in thyroid dysfunction as an irAE [8]. However, biomarkers and susceptible HLA alleles as risk factors in ICIT1DM remain unknown. As ICI-T1DM is a rare disease, investigation of the risk factors has been difficult. In the current study, we have obtained a sufficient number of patients for examination from multiple centers.

In the current study, we aimed to investigate the risk factors in patients with ICI-T1DM, and have identified novel HLA alleles and haplotypes susceptible to ICI-T1DM and possible peripheral blood biomarkers.

\section{METHODS}

\section{Patients}

The study was conducted at the Japanese Red Cross Society Wakayama Medical Center (JRCW), Wakayama Medical University Hospital (WMU), and Nagoya University (NU). Patients with advanced malignant diseases (MM; non-small cell lung cancer [NSCLC]; small cell lung cancer [SCLC]; gastric cancer, renal cell carcinoma, urothelial cancer, hypopharyngeal cancer, and Hodgkin lymphoma) that received ICI treatment were recruited during 2016 to 2021. ICI treatments included anti-PD-1 antibody (nivolumab or pembrolizumab), anti-PD-L1 antibody (durvalumab), or anti-CTLA-4 antibody (ipilimumab) following nivolumab. ICIs were intravenously administered as follows: pembrolizumab, $2 \mathrm{mg} / \mathrm{kg}$ every 3 weeks; nivolumab, 3 $\mathrm{mg} / \mathrm{kg}$ every 2 weeks; durvalumab, 1,500 mg every 3 weeks (until 4 times, then every 4 weeks); ipilimumab following nivolumab, $3 \mathrm{mg} / \mathrm{kg}$ ipilimumab following nivolumab, $2 \mathrm{mg} / \mathrm{kg}$ every 3 weeks. Patients who had infectious diseases or who were treated with glucocorticoids were excluded. The study protocol was approved by the JRCW (No. 782, 891), WMU (No. 1987), and NU (No. UMIN000019024) Institutional Ethical Review Board, and written informed consent was obtained from all participants. All procedures were in accordance with the ethical standards of the responsible committee on human experimentation and with the Declaration of Helsinki, as revised in Fortaleza, Brazil in October 2013.

\section{Assessment of irAEs and ICI-T1DM}

For assessment of irAEs, we used the descriptions and grading scales of National Cancer Institute (NCI) Common Terminology Criteria for Adverse Events version 3.0. The diagnostic criteria for fulminant T1DM (F1DM) are as follows [11]: (1) occurrence of diabetic ketosis or ketoacidosis soon (approximately 7 days) after the onset of hyperglycemic symptoms (elevation of urinary and/or serum ketone bodies at first visit); (2) plasma glucose (PG) level $\geq 288 \mathrm{mg} / \mathrm{dL}$ and hemoglobin A1c (HbAlc) level $<8.7 \%$ at first visit; and (3) urinary C-peptide immunoreactivity (CPR) excretion $<10 \mu \mathrm{g} /$ day or fasting serum CPR level $<0.3$ and $<0.5 \mathrm{ng} / \mathrm{mL}$ after intravenous glucagon loading (or after a meal) at the onset.

The diagnostic criteria for T1DM are as follows [12]: (1) occurrence of diabetic ketosis or ketoacidosis within the first 3 months after the onset of hyperglycemic symptoms (thirst, polydipsia, polyuria, weight loss); (2) need for continuous insulin therapy after the diagnosis of diabetes mellitus (DM); (3) a positive test result for anti-islet autoantibodies; and (4) presence of endogenous insulin deficiency without verifiable anti-islet autoantibodies. Acute-onset T1DM (autoimmune) fulfilled criteria 1, 2, and 3, while acute-onset T1DM fulfilled criteria 1, 2, and 4 .

\section{Data and sample collection}

Baseline characteristics of the patients with ICI-T1DM including the type of cancer, ICI treatments, and the manifestation of ICI-T1DM were recorded (Table 1). The following factors were investigated: serum amylase (AMY), arterial blood gas data, serum beta-hydroxybutyrate (BHB), serum acetoacetic acid (AcAc), serum total ketone body (TKB), urine ketone bodies, serum non-esterified fatty acid (NEFA), anti-pancreatic betacell autoantibodies, occurrences of other irAEs, thyroid autoantibodies, insulin secretion status at the onset and 1 month after, HLA typing tests, tumor response, and continuation of ICI (Table 2). For longitudinal examination, white blood cell (WBC), 


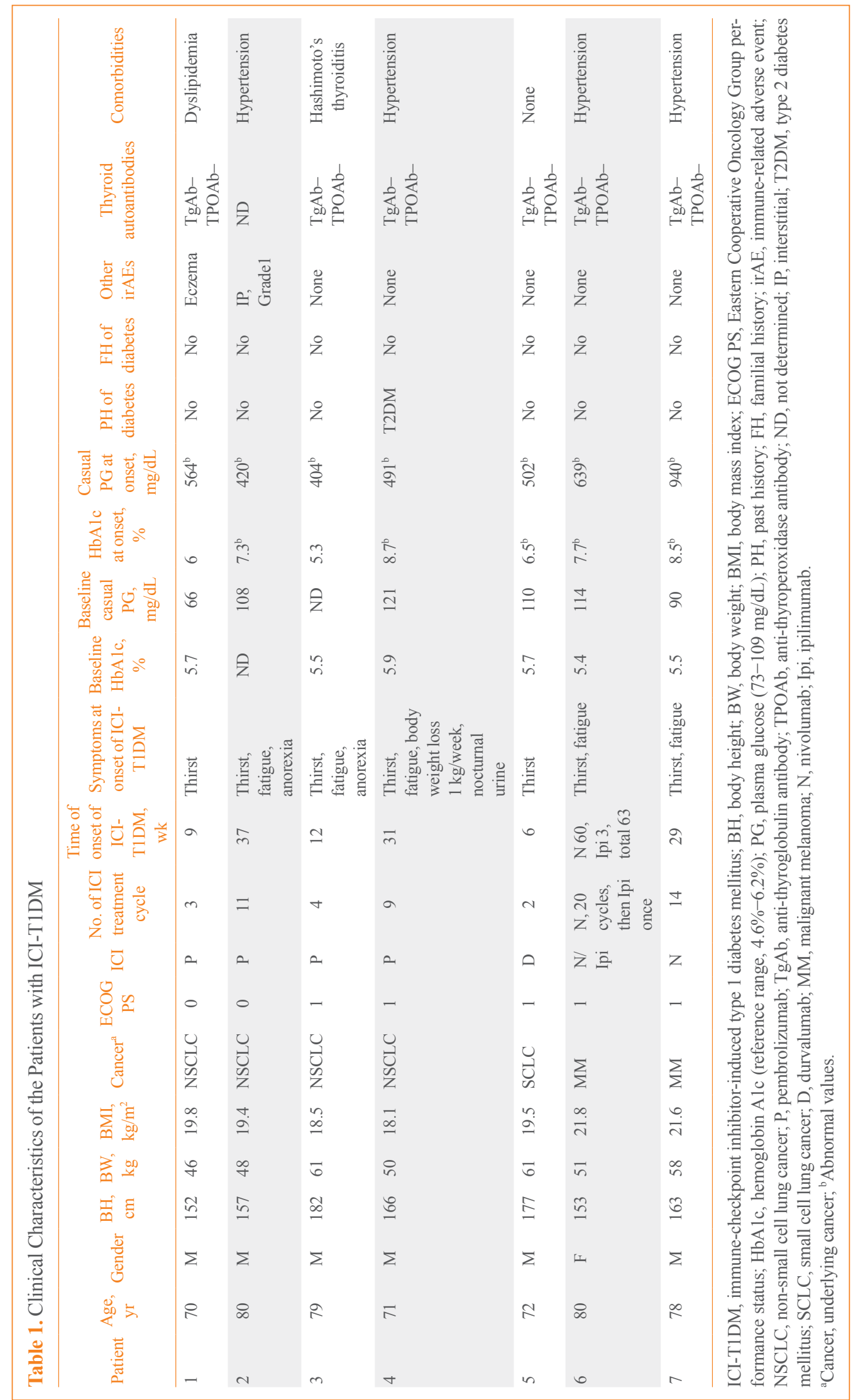


Table 2. Laboratory Investigations of the Patients with ICI-T1DM at Onset

\begin{tabular}{|c|c|c|c|c|c|c|c|c|c|c|c|c|c|c|c|c|}
\hline Patient & $\begin{array}{l}\text { IRI at } \\
\text { onset, } \\
\mu \mathrm{U} / \mathrm{mL}\end{array}$ & $\begin{array}{c}\text { IRI at } 1 \\
\text { month } \\
\text { after the } \\
\text { onset, } \\
\mu \mathrm{U} / \mathrm{mL}\end{array}$ & $\begin{array}{c}\text { Serum } \\
\text { CPR at } \\
\text { onset, } \\
\mathrm{ng} / \mathrm{mL}\end{array}$ & $\begin{array}{l}\text { Serum } \\
\text { CPR at } 1 \\
\text { month } \\
\text { after the } \\
\text { onset, } \\
\text { ng/mL }\end{array}$ & $\begin{array}{l}\text { AMY, } \\
\text { U/L }\end{array}$ & $\begin{array}{l}\text { Blood, } \\
\mathrm{pH}^{\mathrm{a}}\end{array}$ & $\begin{array}{c}\mathrm{BE}, \\
\mathrm{mmol} / \mathrm{L}\end{array}$ & $\begin{array}{l}\mathrm{HCO}_{3}^{-}, \\
\mathrm{mmol} / \mathrm{L}\end{array}$ & $\begin{array}{l}\text { BHB, } \\
\mu \mathrm{mol}\end{array}$ & $\begin{array}{l}\text { AcAc, } \\
\mu \mathrm{mol}\end{array}$ & $\begin{array}{l}\mathrm{TKB} \\
\mu \mathrm{mol}\end{array}$ & $\begin{array}{l}\text { Urine } \\
\text { ketone }\end{array}$ & $\begin{array}{l}\text { NEFA, } \\
\mu \mathrm{Eq} / \mathrm{L}\end{array}$ & $\begin{array}{c}\text { Anti- } \\
\mathrm{GAD} \\
\mathrm{Ab} / \\
\text { Insulin } \\
\mathrm{Ab}\end{array}$ & $\begin{array}{c}\text { Tumor } \\
\text { response }\end{array}$ & $\begin{array}{c}\text { Continuation } \\
\text { of ICI }\end{array}$ \\
\hline 1 & $0.8^{\mathrm{b}}$ & $<0.4^{\mathrm{b}}$ & $0.17^{\mathrm{b}}$ & $<0.02^{\mathrm{b}}$ & 83 & 7.372 & 0 & 24.9 & $818^{\mathrm{b}}$ & $452^{\mathrm{b}}$ & $1,270^{b}$ & Negative & $936^{\mathrm{b}}$ & $\mathrm{Ne} / \mathrm{Ne}$ & PR & Continued \\
\hline 2 & ND & ND & $<0.02^{\mathrm{b}}$ & $<0.02^{\mathrm{b}}$ & 70 & $7.306^{b}$ & $-6.2^{\mathrm{b}}$ & $19.2^{\mathrm{b}}$ & $5,159^{b}$ & $613^{\mathrm{b}}$ & $5,772^{b}$ & $3+^{\mathrm{b}}$ & $2,311^{b}$ & $\mathrm{Ne} / \mathrm{Ne}$ & PR & Continued \\
\hline 3 & 7.7 & $<0.4^{\mathrm{b}}$ & 2.31 & $<0.02^{\mathrm{b}}$ & $228^{b}$ & $\mathrm{ND}$ & $\mathrm{ND}$ & ND & ND & ND & ND & ND & ND & $\mathrm{Ne} / \mathrm{ND}$ & PR & Discontinued \\
\hline 4 & $0.7^{b}$ & $<0.4^{\mathrm{b}}$ & 1.33 & $<0.02^{\mathrm{b}}$ & ND & 7.393 & 0.1 & 24.5 & $1,778^{\mathrm{b}}$ & $583^{\mathrm{b}}$ & $2,361^{\mathrm{b}}$ & $1+^{\mathrm{b}}$ & $1,115^{b}$ & $\mathrm{Ne} / \mathrm{Ne}$ & PR & Continued \\
\hline 5 & $<0.4^{\mathrm{b}}$ & $<0.4^{\mathrm{b}}$ & $<0.02^{\mathrm{b}}$ & $<0.02^{\mathrm{b}}$ & 47 & 7.43 & 6.1 & 30.8 & $2,865^{\mathrm{b}}$ & $1,093^{\mathrm{b}}$ & $3,958^{\mathrm{b}}$ & $4+^{b}$ & $1,028^{b}$ & $\mathrm{Ne} / \mathrm{Ne}$ & PR & Continued \\
\hline 6 & ND & ND & $<0.01^{\mathrm{b}}$ & $<0.01^{\mathrm{b}}$ & 47 & 7.43 & -0.2 & 24.2 & 73.4 & 57.5 & $130.9^{b}$ & $1+^{\mathrm{b}}$ & ND & $\mathrm{Ne} / \mathrm{Po}$ & $\mathrm{CR}$ & Continued \\
\hline 7 & $2.5^{\mathrm{b}}$ & $\mathrm{ND}^{\mathrm{b}}$ & $0.16^{\mathrm{b}}$ & $<0.01^{\mathrm{b}}$ & $137^{\mathrm{b}}$ & $7.234^{b}$ & $-10.9^{\mathrm{b}}$ & $15^{\mathrm{b}}$ & $1,374^{b}$ & $726^{b}$ & $2,100^{\mathrm{b}}$ & $1+^{\mathrm{b}}$ & ND & $\mathrm{Ne} / \mathrm{Ne}$ & $\mathrm{CR}$ & Continued \\
\hline
\end{tabular}

ICI-T1DM, immune-checkpoint inhibitor-induced type 1 diabetes mellitus; IRI, immunoreactive insulin; CPR, C-peptide immunoreactivity; AMY, amylase (44-132 U/L); BE, base excess $(0 \pm 4 \mathrm{mmol} / \mathrm{L}) ; \mathrm{HCO}_{3}{ }^{-}$, bicarbonate $(22-26 \mathrm{mmol} / \mathrm{L}) ; \mathrm{BHB}$, beta-hydroxybutyrate $(<74 \mu \mathrm{mol}) ; \mathrm{AcAc}$, acetoacetic

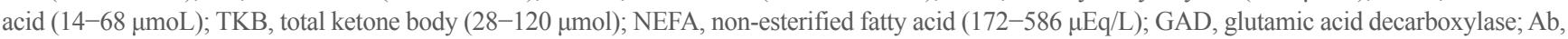
autoantibody; $\mathrm{Ne}$, negative; $\mathrm{PR}$, partial response; ND, not determined; Po, positive; $\mathrm{CR}$, complete response.

${ }^{\mathrm{a}} \mathrm{pH}, 7.35-7.45$; ${ }^{\mathrm{a}}$ Abnormal values.

hemoglobin, platelet count (Plt), absolute neutrophil count (ANC), relative neutrophil count (RNC), absolute lymphocyte count (ALC), relative lymphocyte count, absolute eosinophil count (AEC), relative eosinophil count (REC), absolute basophil count, relative basophil count, absolute monocyte count, relative monocyte count, neutrophil-lymphocyte rate: $\mathrm{RNC/}$ RLC (NLR), derived NLR (dNLR): ANC/(WBC-ALC) [13], neutrophil-eosinophil rate: RNC/REC (NER), platelet-lymphocyte rate: Plt/ALC, albumin (Alb), aspartate aminotransferase (AST), alanine aminotransferase (ALT), alkaline phosphatase (ALP), blood urea nitrogen (BUN), creatinine (Cr), estimated glomerular filtration rate (eGFR), sodium $(\mathrm{Na})$, potassium $(\mathrm{K})$, chloride $(\mathrm{Cl})$, calcium $(\mathrm{Ca})$, lactate dehydrogenase, C-reactive protein, free thyroxine, and thyrotropin were examined. The eGFR was used to estimate kidney function and was calculated as follows [14]: Male: eGFR $\left(\mathrm{mL} / \mathrm{min} / 1.73 \mathrm{~m}^{2}\right)=0.741 \times 175 \times$ age $^{-0.203} \times(\text { serum } \mathrm{Cr})^{-1.154}$; Female: eGFR $=0.741 \times 175 \times$ age ${ }^{-0.203} \times(\text { serum } \mathrm{Cr})^{-1.154} \times 0.742(<90)$.

Peripheral blood samples were collected from patients at baseline and at every visit during ICI treatment ( 0 weeks and at $3 \pm 2$-week intervals). The levels or ratio of each result of the examinations was evaluated as follows: rate of change in values (1) during the first 12 weeks; (2) between baseline ( 0 week) and onset of ICI-T1DM; and (3) during 6 weeks until the onset. Patient 1 was excluded from the analysis because the onset was just 6 weeks after the first ICI treatment. For patients 6 and 7 , cytokines/chemokines were measured at 0 weeks, at 3 weeks (before second treatment), at 3 weeks before the onset of ICI$\mathrm{T} 1 \mathrm{DM}$, and then at the onset. Serum samples were centrifuged at $3,000 \mathrm{rpm}$ at $4^{\circ} \mathrm{C}$ for 15 minutes and stored at $-80^{\circ} \mathrm{C}$ until measurement.

\section{Cytokine and chemokine analysis}

Cytokine and chemokine levels were measured as previously described using Bio-Plex Pro Human 17-plex panels (interleukin [IL]-1 $\beta$, IL-2, IL-4, IL-5, IL-6, IL-7, IL-8, IL-10, IL-12, IL13 , IL-17, interferon [IFN]- $\gamma$, tumor necrosis factor- $\alpha$, granulocyte-colony stimulating factor [G-CSF], granulocyte-macrophage-colony stimulating factor [GM-CSF], monocyte chemoattractant protein [MCP]-1, macrophage Inflammatory protein [MIP]-1 $\beta$ ) (Bio-Rad Laboratories, Hercules, CA, USA) [7]. Each assay was performed in duplicate. Absolute fluorescence intensity $(\mathrm{Fi})$ values were derived from the discovery assay and are in direct proportion to reflect the number of proteins in the samples, and Fi values were $\log 2$ transformed for analysis. Fi values were used for relative comparisons of each patient or timing in the current study. For the comparison of controls, the results of cytokine/chemokine levels in the 13 patients with thyroid irAE (IR) and 13 patients without any irAEs (non-IR) in the previous report [7] were presented.

\section{HLA-genotyping}

DNA was extracted from blood, and genotyping for the HLAA, B, C, DRB1, DRB345, DQA1, DQB1, DPA1, and DPB1 al- 
leles was carried out by the next-generation sequencing method (GenoDive Pharma, Kanagawa, Japan). Haplotype frequencies were estimated by direct counting [15]. The allele and haplotype frequencies were compared to those of non-IR with ICI therapy (referred to as ICI-controls) [7], and those of healthy Japanese controls $[16,17]$. In a confirmatory set, HLA-DPB1 was genotyped using a Luminex system with WAKFlow HLA typing kits (Wakunaga Pharmaceutical, Hiroshima, Japan), as described previously [18].

\section{Statistical analysis}

Values among more than two groups were ascertained by the Kruskal-Wallis test, followed by the Wilcoxon signed-rank test with Bonferroni correction, in which each value was compared with other values in the time course. Association of allele or haplotype frequencies was analyzed using Fisher's exact test with $2 \times 2$ contingency tables. Statistical analyses were performed using JMP version 15 (SAS Institute Inc., Cary, NC, USA). $P$ values $<0.05$ were considered to be statistically significant.

\section{RESULTS}

\section{Clinical characteristics of patients with ICI-T1DM}

A total of 871 (432 in JRCW and 439 in WMU) patients with malignant diseases treated with ICIs were screened, and seven $(0.8 \%)$ patients developed ICI-T1DM (four patients with NSCLC and a patient with SCLC in JRCW and two patients with MM in WMU) (Table 1). It should be noted that part of the clinical profile of patient 6 on admission was previously described [4].

In the baseline demographic data (Table 1), male predominance was shown, and ICI treatment until the onset ranged between 2 and 21 cycles. Hyperglycemic symptoms, such as thirst or fatigue, were seen at the onset. Baseline levels of $\mathrm{HbA1c}$ and casual PG were within normal range, but casual PG levels were above $400 \mathrm{mg} / \mathrm{dL}$ and HbA1c levels were $\leq 8.7 \%$ at the onset. Patient 4 had pre-existing type 2 DM. Eczema (patient 1) and interstitial pneumonitis (patient 2) occurred. Thyroid autoantibodies were not detected in five patients tested. Serum immunoreactive insulin (IRI) levels were low in five patients tested (ID $1,3,4,5$, and 7), and casual serum CPR levels were also below the reference range in five patients (ID 1, 2, 5, 6, and 7) at the onset (Table 2). In the course, fasting IRI levels became undetectable in four patients (ID 1, 3, 4, and 5), and serum CPR levels in all seven patients were also undetectable 1 month after the onset (Table 2). The changes in casual PG are shown in Fig. 1.
Of note, two patients (ID 3 and 7) exhibited elevation in serum AMY levels at the onset, and subsided (Table 2). Patients 2 and 7 had metabolic acidosis. Serum BHB and AcAc levels increased in five patients (ID 1, 2, 4, 5, and 7), and TKB levels increased in six patients (ID 1, 2, 4, 5, 6, and 7). Urine ketone bodies were positive in five patients (ID 2, 4, 5, 6, and 7), and negative in patient 1 . Therefore, serum or urine ketone bodies were positive in all six patients tested. NEFA levels increased in four patients (ID 1, 2, 4, and 5). Partial or complete tumor responses were archived in the patients. Pancreatic beta-cell autoantibodies were all negative except for patient 6 , who had a positive conversion of anti-insulin autoantibody at the onset.

\section{Assessment of ICI-T1DM}

Referring to the criteria for F1DM [11]. (1) Occurrence of diabetic ketosis or ketoacidosis was seen in six patients (ID 1, 2, 4, 5 , 6, and 7); (2) PG level $\geq 288 \mathrm{mg} / \mathrm{dL}$ was confirmed in all patients and an $\mathrm{HbA} 1 \mathrm{c}$ level $<8.7 \%$ was observed in six patients; (3) Fasting serum CPR was $<0.3 \mathrm{ng} / \mathrm{mL}$ in five patients (ID 1, 2, 5,6 , and 7) at the onset, and then in all patients 1 month later. Also, referring to the criteria for T1DM [12], all patients tested

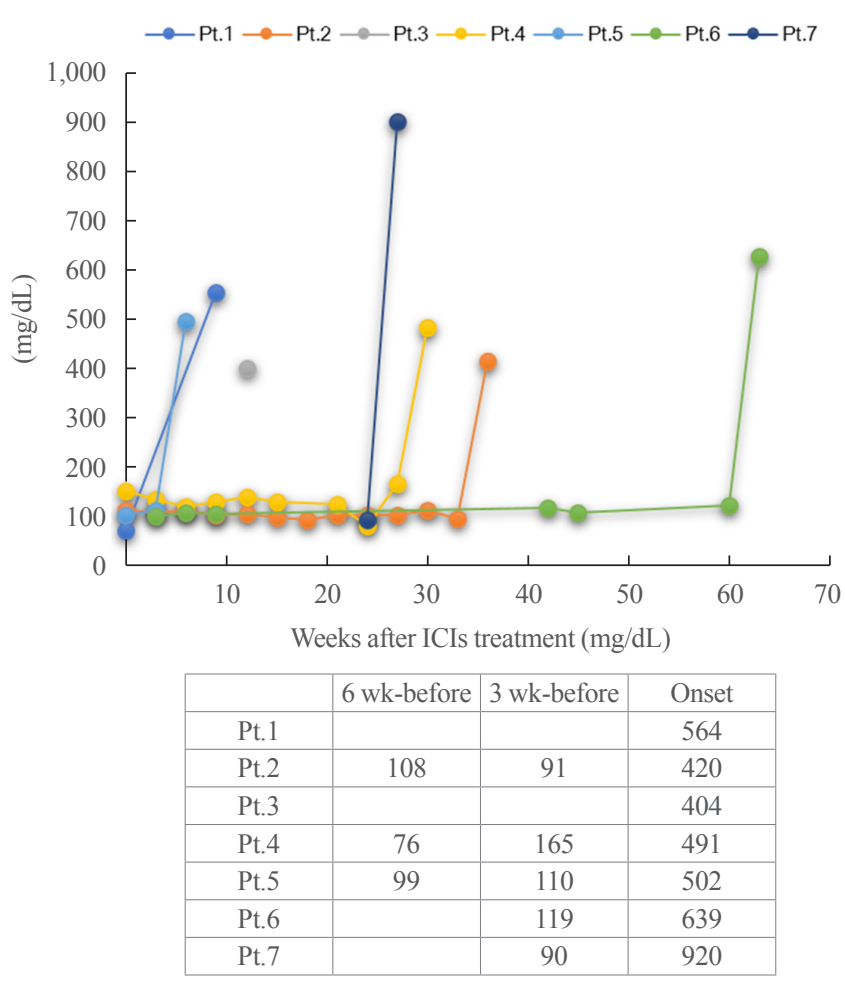

Fig. 1. (A) Glycemic changes in patients with immune-checkpoint inhibitor (ICI)-induced type 1 diabetes mellitus are shown. (B) Casual plasma glucose levels are indicated with changes in the last 6 weeks until the onset. 
were considered to have T1DM (patient 6 had 'autoimmunity').

\section{HLA typing results analysis}

In the HLA allele analysis (Supplemental Tables S1-S13), HLA$C * 01: 02$ and HLA-DRB1*04:05 allele frequencies were significantly higher in ICI-T1DM patients than in controls $(P=0.035$, and $P=0.045$, respectively) (Supplemental Tables S3, S4). Notably, the frequencies of HLA-DPA $1 * 02: 02$ and its associated allele, DPB1*05:01, were significantly associated with an increased risk of ICI-T1DM when compared to controls $(P=0.021$ and $P=0.0075$, respectively) (Supplemental Tables S8, S9).

Confirmatory analysis of HLA-DRB1 and DPB1 alleles in

Table 3. Summary of HLA Alleles and Haplotypes of the Patients with ICI-T1DM

\begin{tabular}{|c|c|c|c|c|c|c|c|c|c|}
\hline \multirow{3}{*}{ Patient } & \multicolumn{9}{|c|}{ HLA alleles or haplotypes } \\
\hline & $\mathrm{C}$ & DRB1 & DQB1 & DPA1 & DPB1 & DRB1-DQB1 & DPA1-DPB1 & DRB1-DC & QB1-DPB1 \\
\hline & $* 01: 02$ & $* 04: 05$ & $* 04: 01$ & $* 02: 02$ & $* 05: 01$ & $* 04: 05-* 04: 01$ & $* 02: 02-* 05: 01$ & $* 04: 05-* 04: 01-* 05: 01$ & $* 08: 03-* 06: 01-* 05: 01$ \\
\hline 1 & & 1 & 1 & 2 & 2 & 1 & 2 & 1 & \\
\hline 2 & 2 & 1 & 1 & 1 & 1 & 1 & 1 & 1 & \\
\hline 3 & & & & 1 & 1 & & 1 & & \\
\hline 4 & 2 & 1 & 1 & 1 & 1 & 1 & & & \\
\hline 5 & 1 & 1 & 1 & 2 & 2 & 1 & 2 & 1 & 1 \\
\hline 6 & & 1 & 1 & 2 & 2 & 1 & 2 & 1 & \\
\hline 7 & 1 & & & 2 & 2 & & 2 & & 2 \\
\hline
\end{tabular}

Numbers of alleles or haplotypes in patients with ICI-T1DM are shown. The alleles or haplotypes that significantly increased from controls are represented.

HLA, human leukocyte antigen; ICI-T1DM, immune-checkpoint inhibitor-induced type 1 diabetes mellitus.

Table 4. Summary of HLA Alleles and Haplotypes of the Patients with ICI-T1DM

\begin{tabular}{|c|c|c|c|c|c|c|c|c|c|}
\hline \multirow{2}{*}{ HLA alleles or haplotypes } & $\mathrm{C}$ & DRB1 & DQB1 & DPA1 & DPB1 & $\begin{array}{r}\text { DRB1- } \\
\text { DQB1 }\end{array}$ & $\begin{array}{l}\text { DPA1- } \\
\text { DPB1 }\end{array}$ & \multicolumn{2}{|c|}{ DRB1-DQB1-DPB1 } \\
\hline & $* 01: 02$ & $* 04: 05$ & *04:01 & *02:02 & $* 05: 01$ & $\begin{array}{l}* 04: 05- \\
\quad * 04: 01\end{array}$ & $\begin{array}{l}* 02: 02- \\
\quad * 05: 01\end{array}$ & $\begin{array}{c}* 04: 05-* 04: 01 \\
* 05: 01\end{array}$ & $\begin{array}{l}08: 03-* 06: 01- \\
\quad * 05: 01\end{array}$ \\
\hline Controls, $\%^{\mathrm{a}}$ & 17.3 & 13.4 & 12.9 & 43.5 & 38.4 & 12.8 & 34.3 & 7.32 & 3.54 \\
\hline $\begin{array}{l}\% \text { of alleles or haplotypes in } \\
\text { ICI-T1DM patients (total } 14 \text { ) }\end{array}$ & 42.9 & 35.7 & 35.7 & 78.6 & 78.6 & 35.7 & 71.4 & 4 & 3 \\
\hline $\begin{array}{l}\text { No. of alleles or haplotypes in } \\
\text { ICI-T1DM patients (total 14) }\end{array}$ & 6 & 5 & 5 & 11 & 11 & 5 & 10 & 28.6 & 21.4 \\
\hline$P$ value $^{\mathrm{b}}$ & $0.035^{\mathrm{d}}$ & $0.045^{\mathrm{d}}$ & $0.045^{\mathrm{d}}$ & $0.021^{\mathrm{d}}$ & $0.0075^{\mathrm{d}}$ & $0.045^{\mathrm{d}}$ & $0.016^{\mathrm{d}}$ & $0.029^{\mathrm{d}}$ & $0.039^{\mathrm{d}}$ \\
\hline OR & 3.66 & 3.72 & 3.72 & 4.67 & 5.98 & 3.72 & 4.85 & 5.31 & 6.55 \\
\hline $95 \% \mathrm{CI}$ & $1.13-11.92$ & $1.08-12.83$ & $1.08-12.83$ & $1.23-17.76$ & $1.57-22.82$ & $1.08-12.83$ & $1.42-16.62$ & $1.32-21.36$ & $1.29-33.13$ \\
\hline $\begin{array}{l}\text { No. of alleles or haplotypes in } \\
\text { ICI-controls (total 26) }\end{array}$ & 6 & 4 & 4 & 10 & 7 & 6 & 7 & 2 & 1 \\
\hline$P$ value ${ }^{\mathrm{c}}$ & NS & NS & NS & $0.022^{\mathrm{d}}$ & $0.0027^{d}$ & NS & $0.0093^{\mathrm{d}}$ & NS & NS \\
\hline OR & NA & NA & NA & 5.87 & 9.95 & NA & 6.79 & NA & NA \\
\hline $95 \% \mathrm{CI}$ & NA & NA & NA & $1.31-26.33$ & $2.13-46.56$ & NA & $1.60-28.86$ & NA & NA \\
\hline
\end{tabular}

HLA, human leukocyte antigen; ICI-T1DM, immune-checkpoint inhibitor-induced type 1 diabetes mellitus; OR, odds ratio; CI, confidence interval; NS, not significant; NA, not applicable.

${ }^{a}$ The frequencies in control subjects are shown to the first decimal place. Control subjects for allele analysis: Japanese Society for Histocompatibility and Immunogenetics (http://jshi.umin.ac.jp/standarization/file/JSHI-hyokiallele-2021list.pdf) (JSHI2022) [16], and for haplotype analysis: HLA Laboratory, Japan INC (http://hla.or.jp/med/frequency_search/ja/haplo/) [17]; ${ }^{\mathrm{b}} \mathrm{P}$ value in comparison to the controls in Japanese population [16,17]; ${ }^{\mathrm{c}} P$ value in comparison to the controls with ICI treatment [7]; ${ }^{\mathrm{d}} P$ values less than 0.05 . 
four patients with ICI-T1DM in NU showed increased frequencies of DRB1*04:05 (two of eight alleles, and two carriers in four patients), as well as increased frequencies of DPB1*05:01 (five of eight alleles, and three carriers in four patients). The data of two out of these four patients were previously reported [19].

Next, HLA-DRB1*04:05-DQB1*04:01 and HLA-DPA1*02: 02-DPB $1 * 05: 01$ haplotype frequencies were significantly higher in patients with ICI-T1DM than in controls $(P=0.045$, and $P=0.016$, respectively) (Supplemental Tables S11, S12). HLADRB1*04:05-DQB1*04:01-DPB1*05:01 and HLA-DRB1*08: 03-DQB1*06:01-DPB1*05:01 haplotype frequencies were significantly higher in patients with ICI-T1DM than in controls ( $P=0.029$ and $P=0.039$, respectively) (Supplemental Table S13).

In the comparison of patients with ICI-T1DM and ICI-controls, HLA-DPA1*02:02, DPB1*05:01, and HLA-DPA1*02: 02-DPB $1 * 05: 01$ haplotype frequencies were significantly more frequent in patients with ICI-T1DM than in ICI-controls $(P=$ $0.022, P=0.0027$, and $P=0.0093$, respectively) (Supplemental
Tables S8, S9, S12).

Alleles and haplotypes of seven patients in the initial analysis are summarized (Tables 3, 4). Remarkably, HLA-DPA1*02:02 and DPB $1 * 05: 01$ were present in all patients with ICI-T1DM, and HLA-DPA1*02:02-DPB1*05:01 was found in all but one.

\section{Rate of change in values for each factor after the initiation of ICI treatment}

During the 12 weeks after the initiation of ICI treatment, the levels of some factors changed (Supplemental Table S14), although the $P$ values were not significant after Bonferroni correction.

\section{Rate of change in values for each factor at onset of ICI- T1DM}

In the comparison of the levels in each factor at the onset with those at baseline, a decrease in Plt levels and an increase in levels of RNC, Alb, ALP, BUN, Cr, and K were seen (Supplemen-
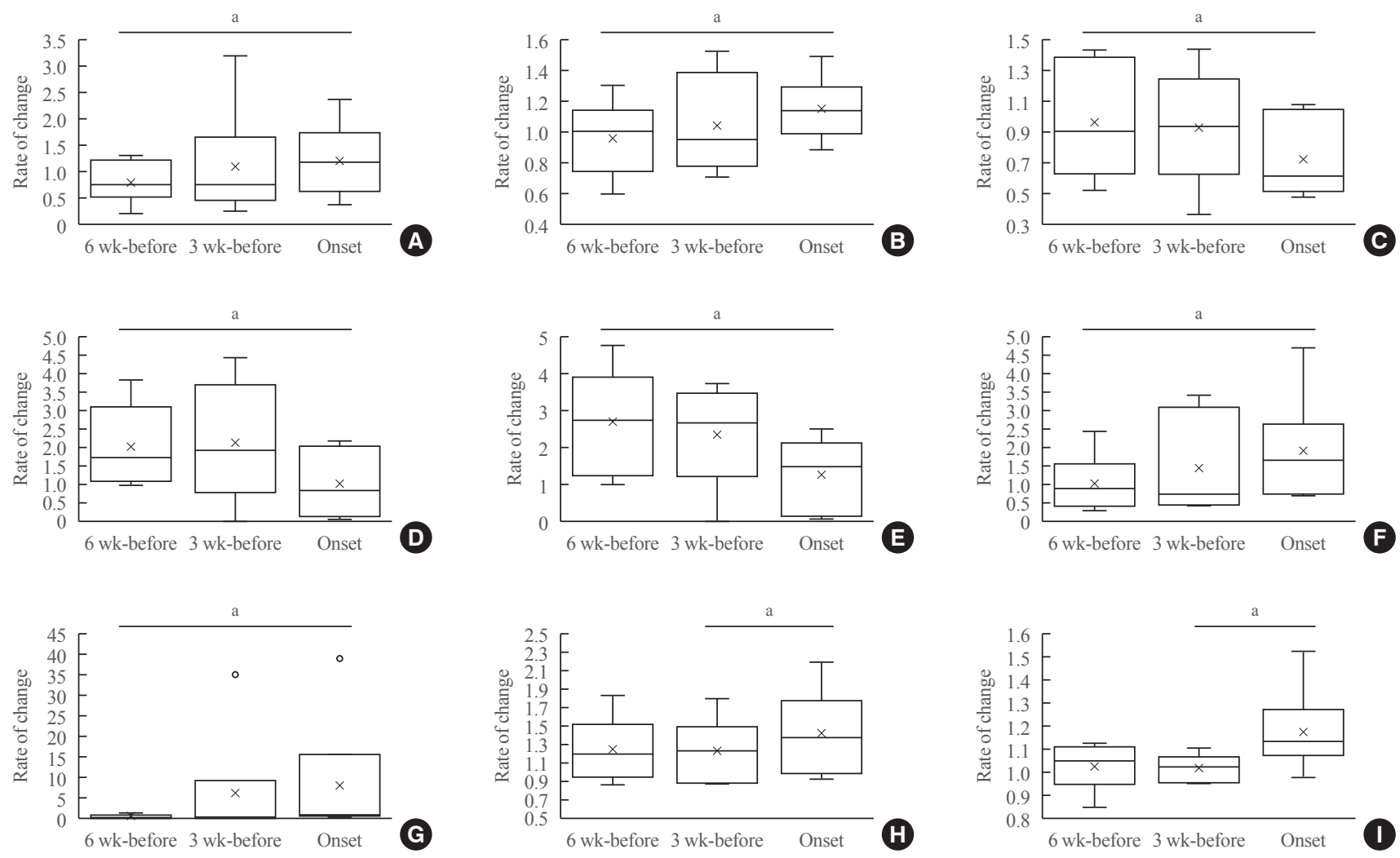

Fig. 2. Rate of changes in values for each factor for 6 weeks until the onset of immune-checkpoint inhibitor-induced type 1 diabetes mellitus. In box-and-whisker plots, minimum, lower quartile, median, upper quartile, and maximum levels are shown as indicated. Average levels are shown as 'x.' The rate of change in values (each value/baseline value: 0 week), and shown on the y-axis. A significant change between 6 weeks before and at the onset was seen in levels of (A) absolute neutrophil count, (B) relative neutrophil count, (C) absolute lymphocyte count, (D) absolute eosinophil count, (E) relative eosinophil count, (F) neutrophil-lymphocyte rate, and (G) neutrophil-eosinophil rate. A significant change between 3 weeks before and at the onset was observed in levels of (H) albumin, and (I) potassium (K). ${ }^{a} P<0.05$. 
tal Table S15).

\section{Rate of change in values for each factor for 6 weeks until the onset of ICI-T1DM}

A longitudinal analysis showed significant increases in ANC, RNC, NLR, NER, and decreases in ALC, AEC, and REC from the levels at 6 weeks before the onset (Fig. 2, Supplemental Table S16). The levels in Alb and K significantly increased from 3 weeks before the onset.

\section{Cytokine and chemokine levels}

The levels in cytokines/chemokines tended to decrease 3 weeks before the onset (the average ratio of onset/3 weeks before was 0.94 in patient 6, and 0.90 in patient 7) (Supplemental Material S1). The levels of Th1 (IL-12, IFN- $\gamma$ ) and Th2 (IL-4, IL-5, and IL-13) cytokines, IL-8, and G-CSF decreased at the onset (both the rate of change $<0.94$ in patient 6 , and that $<0.90$ in patient 7). The levels of MCP-1 and MIP1- $\beta$ were unchanged.

\section{DISCUSSION}

The main goal of this study was to identify risk factors in patients with ICI-T1DM. We revealed that the HLA-DPA1*02:02 and DPB $1 * 05: 01$ alleles were observed in most of the patients, and the allele frequencies were significantly higher than those in ICI-controls and also controls of the Japanese general population. HLA-DRB1*04:05 allele frequencies were significantly higher than those in the general population, but when compared to ICI-controls. Confirmatory analysis for HLA-DPB1*05:01 and DRB1*04:05 alleles further supported the idea that these HLA alleles may confer susceptibility to ICI-T1DM. In addition, we showed that the levels of several blood markers were altered from baseline and during the last 6 weeks until the onset in the longitudinal analysis. These factors may be utilized as biomarkers of ICI-T1DM.

The prevalence of ICI-T1DM $(0.8 \%)$ was close to that in the previous report by Stamatouli et al. [20], in which the incidence was estimated to be $0.9 \%$. The male predominance and tumor variance differed from the findings of Takada et al. [10], who reported that female sex and having $\mathrm{MM}$ were risk factors for the development of ICI-T1DM.

All patients were diagnosed with T1DM [12]. Referring to the criteria for F1DM [11], not all the patients met the criteria for F1DM at onset, but all patients then fulfilled the criteria 1 month later. Taken together, ICI-T1DM is considered to be a mixed type of T1DM and F1DM. The levels of HbA1c and ca- sual PG acutely increased at the onset of ICI-T1DM; therefore, routine monitoring of $\mathrm{HbA} 1 \mathrm{c}$ and $\mathrm{PG}$ is recommended for an earlier diagnosis of ICI-T1DM.

Hernando-Calvo et al. [21] reported that patients with MM switching from high dNLR to low dNLR had significantly better overall survival at the end of cycle 2 of ICI treatment. Jiang et al. [22] reported that patients with low Alb before ICI therapy had a significantly increased risk of progression in patients with NSCLC. In the current study, dNLR rose at week 9, and Alb increased at week 6 , but the reason was unknown, and all patients had a favorable prognosis. In a comparison of the levels of each factor at the onset to those at baseline, Fujisawa et al. [9] reported that Grade 3 or 4 irAEs and lung/GI irAEs were associated with increased WBC and decreased RLC between baseline and onset of irAEs. ICI-T1DM as a systemic irAE may therefore be related to alteration in peripheral blood cell counts.

Mertoglu and Gunay [23] reported that NLR significantly increased in patients with type 2 DM. They suggested that NLR represents the status of inflammation, which plays a central role in the development of type 2 DM. In the current study, the increase in ANC, RNC, and NLR, and the decrease in ALC from 6 weeks before the onset are considered to be associated with the development of ICI-T1DM. The increase in NER and the decrease in AEC and REC may be additional unique findings to ICI-T1DM observed in the current study. The increased levels in Alb and $\mathrm{K}$ at onset from 3 weeks before may represent dehydration and alteration in metabolism by DM. However, since there is no comparison with those who have not developed ICIT1DM, these significances need further investigation.

In the current study, HLA-DPA1*02:02 and DPB1*05:01 were observed in most patients with ICI-T1DM, and their allele or haplotype frequencies were significantly increased. HLADPB $1 * 05: 01$ was confirmed to be important in four additional patients with ICI-T1DM. HLA-DPB1*05:01 seemed to be related to HLA-DRB1*04:05, HLA-DRB1*09:01, and HLADRB1*08:03 [6,8]. It was therefore summarized that (1) HLADPA1*02:02 and DPB1*05:01 may be strongly related to the immune reaction in ICI-T1DM, followed by (2) DRB1*04:05, (3) DRB1*08:03, and (4) C*01:02. These HLA alleles or haplotypes are different from those observed in thyroid irAE [8], and pituitary irAE [6].

Erlich et al. [24] found that HLA-DRB1*03:01, DRB1*04: 05, DRB1*04:01, and DRB1*04:02 alleles confer susceptibility, in contrast, the DRB1*15:01 allele is protective against T1DM. Kawabata and Ikegami [25] reported that DRB1*04:05 and DRB1*09:01 were associated with F1DM and T1DM in Asia. 
The importance of DRB1*04:05 in ICI-T1DM has been suggested [20]. HLA-DRB1 typing results of the patients with ICIT1DM in Japan are as follows: DRB1*11:01/*13:02 [26], DRB1*04:05 [27], DRB1*04:05, and DRB1*09:01 in another [28], and DRB1*04:05/*04:06 [29]. DRB1*04:05 allele increase was also observed in the current study. Interestingly, Tsang et al. [30] reported that some patients with ICI-T1DM possessed T1DM-protective HLA-DRB1 alleles. de Filette et al. [31] mentioned that in addition to the genotypes susceptible to T1DM/F1DM (such as DR3, DR4, or DR9), protective ones were also seen in patients with ICI-T1DM. From these reports, it is speculated that ICI-T1DM may constitute a distinct pathophysiologic entity from T1DM/F1DM. HLA-DRB1*15:01DQB1*06:02 was more frequently seen in ICI-controls than in controls in the general population (Supplemental Table S13). Although the reason is unclear, considering that the haplotype is generally scarce [17], a certain association of HLA with the occurrence of malignancies was suggested.

Notably, HLA-DPB1*02:01 was shown to confer disease susceptibility to childhood-onset T1DM in a Japanese population [32], and HLA-DPA1*01:03-DPB1*03:01/*02:02 has been reported to be associated with susceptibility to T1DM in Caucasians [33]. These alleles and haplotypes were not associated with ICI-T1DM in the current study. The predominance of the HLA-DPA1*02:02-DPB1*05:01 haplotype in ICI-T1DM is therefore a unique finding, and these alleles and haplotypes may have a critical role in disease promotion. HLA-DPB1*05:01 allele is also one of the risk alleles in Graves' disease in the Japanese population [34].

We could not confirm the increase of HLA-C*01:02-related haplotypes such as HLA-C*01:02-DRB1*04:05-DQB1*04:01DPB $1 * 05: 01$ because of the difficulty of haplotype identification. Therefore, it is unclear that whether the increase in HLA$C^{*} 01: 02$ is due to its related haplotypes or other factors.

The most well-known role of the HLA molecule is to bind and present epitopes to pathogenic T-cells [35]. Autoantigen epitope peptides are presented with HLA on the surface of antigen-presenting cells in autoimmune diseases [35].

Pancreatic beta-cell autoantigen, as well as tumor-associated antigen/neoantigen, could be presented with HLA on the surface of antigen-presenting cells $[4,6,8,35]$. We speculate that autoimmune disease-predisposing HLA may also be involved with malignant diseases. The HLA alleles and haplotypes identified in the current study may be useful for the prediction of ICIT1DM. Yoneda et al. [36] investigated the pancreas of a patient with renal cell carcinoma who developed T1DM with ICI treat- ment. Infiltration of CD8+ T-cells in the pancreas was found, and it was concluded that the low levels of PD-L1 expression in the pancreatic islet might be associated with beta-cell injury [36]. Importantly, antibodies such as an anti-glutamic acid decarboxylase antibody or anti-insulin antibody are often undetectable in patients with ICI-T1DM, possibly reflecting acute damage of beta cells, as in the current study [30]. The development of anti-insulin autoantibodies in patient 6 and elevation of serum AMY in two patients suggest that autoimmunity and/or inflammation may be involved in the development of ICIT1DM.

Although the sample size was small and the comparisons were relative, the results of cytokine/chemokine measurements were interesting. Th2 cytokines (IL-4, IL-5, and IL-13) have been reported to be protective for the development of T1DM [37]. The decrease in Th2 cytokines at the onset of ICI-T1DM in the current study is consistent with previous findings in T1DM. Th1 cytokines (IFN- $\gamma$ ) have been reported to be associated with inducing T1DM, but also with suppressing diabetogenic CD8+ Tcells [37]. Although Th1 functions are known to be predominant in the development of T1DM [37], the decrease in Th1 cytokines in the current study might therefore uniquely contribute to the development of ICI-T1DM; however, the pathogenic significance is unclear. Purohit et al. [38] reported that the levels of serum IL-8, IL-1Ra, MCP-1, and MIP1- $\beta$ decreased in patients with T1DM. The decreased serum IL-8 level and the unchanged levels of MCP-1, and MIP1- $\beta$ at the onset in the two patients suggest specific cytokine/chemokine profiles in ICI-T1DM. In comparison to the earlier study on the cytokine/chemokine in the development of thyroid irAE [7], the levels of IL-1 $\beta$, IL-2, IL-8, G-CSF, GM-CSF, and MCP-1 slightly changed or remained unchanged between baseline and 3 weeks after ICI treatment; therefore, the alterations of cytokine/chemokine in ICI-T1DM seemed to be different from those in thyroid irAEs.

Hong et al. [39] reported four patients with ICI-T1DM. They showed that all patients showed antitumor responses after ICI therapy, which is consistent with our results. Due to the small sample size because of the rarity of this disorder, protective alleles, such as DRB1*15:01, in T1DM could not be identified in ICI-T1DM [24], and it is still unclear how each HLA risk allele and the corresponding haplotypes contribute to the development of ICI-T1DM. Moreover, statistical techniques such as multiple regression analysis to identify the association of the HLA risk alleles and haplotypes with blood biomarkers could not be conducted because of the insufficient number of patients. Further investigations with a greater number of cases are required to 
clarify the precise mechanisms, biomarkers, and correlations with antitumor activities in ICI-T1DM.

In conclusion, novel susceptible HLA alleles and haplotypes to ICI-T1DM were identified. HLA typing in combination with peripheral blood markers may be utilized as biomarkers in ICIT1DM.

\section{CONFLICTS OF INTEREST}

Shintaro Iwama received personal fees from Ono Pharmaceutical Co. Ltd., Bristol-Myers Squibb, Chugai Pharmaceutical Co. Ltd., and MSD K.K. outside of this study.

Hiroshi Arima received grants from Ono Pharmaceutical Co. Ltd., MSD K.K., and Chugai Pharmaceutical Co. Ltd., and personal fees from Ono Pharmaceutical Co. Ltd., Bristol-Myers Squibb, and MSD K.K. outside of this study. The other authors declare no conflicts of interest.

\section{ACKNOWLEDGMENTS}

We are indebted to Dr. Hidetoshi Inoko (Tokai University) and Yuko Okudaira (GenoDive Pharma Inc.) for technical advice. We acknowledge proofreading and editing by Benjamin Phillis at the Clinical Study Support Center, Wakayama Medical University.

This work was partially supported by the Takeda Science Foundation, and Grant-in-Aid for Scientific Research from the Ministry of Education, Science, Sports, and Culture, Japan (20K17541 and 21K08544).

\section{AUTHOR CONTRIBUTIONS}

Conception or design: H.I. Acquisition, analysis, or interpretation of data: H.I., Y.K., S.I., T.H., G.I., T.S., Y.Y., M.J., H.K., T.K., S.I., H.A., T.M. Drafting the work or revising: H.I., Y.K., S.I., T.H., G.I., T.S., Y.Y., M.J., H.K., T.K., S.I., H.A., T.M. Final approval of the manuscript: H.I., Y.K., S.I., T.H., G.I., T.S., Y.Y., M.J., H.K., T.K., S.I., H.A., T.M.

\section{ORCID}

Hidefumi Inaba https://orcid.org/0000-0002-7718-734X

\section{REFERENCES}

1. Blank CU, Enk A. Therapeutic use of anti-CTLA-4 antibod-

Copyright $(\odot 2022$ Korean Endocrine Society ies. Int Immunol 2015;27:3-10.

2. Okazaki T, Honjo T. PD-1 and PD-1 ligands: from discovery to clinical application. Int Immunol 2007;19:813-24.

3. Arima H, Iwama S, Inaba H, Ariyasu H, Makita N, Otsuki $\mathrm{M}$, et al. Management of immune-related adverse events in endocrine organs induced by immune checkpoint inhibitors: clinical guidelines of the Japan Endocrine Society. Endocr J 2019;66:581-6.

4. Shiba M, Inaba H, Ariyasu H, Kawai S, Inagaki Y, Matsuno $\mathrm{S}$, et al. Fulminant type 1 diabetes mellitus accompanied by positive conversion of anti-insulin antibody after the administration of anti-CTLA-4 antibody following the discontinuation of anti-PD-1 antibody. Intern 2018;57:2029-34.

5. Inaba H, Ariyasu H, Takeshima K, Iwakura H, Akamizu T. Comprehensive research on thyroid diseases associated with autoimmunity: autoimmune thyroid diseases, thyroid diseases during immune-checkpoint inhibitors therapy, and immunoglobulin-G4-associated thyroid diseases. Endocr J 2019;66:843-52.

6. Inaba H, Ariyasu H, Iwakura H, Ueda Y, Kurimoto C, Uraki $\mathrm{S}$, et al. Comparative analysis of human leucocyte antigen between idiopathic and anti-PD-1 antibody induced isolated adrenocorticotropic hormone deficiency: a pilot study. Clin Endocrinol (Oxf) 2019;91:786-92.

7. Kurimoto C, Inaba H, Ariyasu H, Iwakura H, Ueda Y, Uraki $\mathrm{S}$, et al. Predictive and sensitive biomarkers for thyroid dysfunctions during treatment with immune-checkpoint inhibitors. Cancer Sci 2020;111:1468-77.

8. Inaba H, Ariyasu H, Iwakura H, Kurimoto C, Takeshima K, Morita S, et al. Distinct clinical features and prognosis between persistent and temporary thyroid dysfunctions by immune-checkpoint inhibitors. Endocr J 2021;68:231-41.

9. Fujisawa Y, Yoshino K, Otsuka A, Funakoshi T, Fujimura T, Yamamoto Y, et al. Fluctuations in routine blood count might signal severe immune-related adverse events in melanoma patients treated with nivolumab. J Dermatol Sci 2017; 88:225-31.

10. Takada S, Hirokazu H, Yamagishi K, Hideki S, Masayuki E. Predictors of the onset of type 1 diabetes obtained from realworld data analysis in cancer patients treated with immune checkpoint inhibitors. Asian Pac J Cancer Prev 2020;21:16979.

11. Imagawa A, Hanafusa T, Awata T, Ikegami H, Uchigata $Y$, Osawa H, et al. Report of the Committee of the Japan Diabetes Society on the research of fulminant and acute-onset type 1 diabetes mellitus: new diagnostic criteria of fulminant

www.e-enm.org 
type 1 diabetes mellitus (2012). J Diabetes Investig 2012;3: 536-9.

12. Kawasaki E, Maruyama T, Imagawa A, Awata T, Ikegami H, Uchigata $\mathrm{Y}$, et al. Diagnostic criteria for acute-onset type 1 diabetes mellitus (2012): report of the Committee of Japan Diabetes Society on the research of fulminant and acute-onset type 1 diabetes mellitus. J Diabetes Investig 2014;5:115-8.

13. Capone M, Giannarelli D, Mallardo D, Madonna G, Festino L, Grimaldi AM, et al. Baseline neutrophil-to-lymphocyte ratio (NLR) and derived NLR could predict overall survival in patients with advanced melanoma treated with nivolumab. J Immunother Cancer 2018;6:74.

14. Matsuo S, Imai E, Horio M, Yasuda Y, Tomita K, Nitta K, et al. Revised equations for estimated GFR from serum creatinine in Japan. Am J Kidney Dis 2009;53:982-92.

15. Imanishi T, Akaza T, Kimura A, Tokunaga K, Gojobori T. Allele and haplotype frequencies for HLA and complement loci in various ethnic groups. In: Tsuji K, Aizawa M, Sasazuki T. HLA 1991: Proceedings of the Eleventh International Histocompatibility Workshop and Conference; 1991 Nov 6-13; Yokohama, JP. Oxford: Oxford University Press; 1992. p. 1065-220.

16. Japanese Society for Histocompatibility and Immunogenetics. List of estimated HLA alleles 2021 [Internet]. Tokyo: JSHI; 2022 [cited 2022 Jan 21]. Available from: http://jshi. umin.ac.jp/standarization/file/JSHI-hyokiallele-2021list. pdf.

17. HLA Laboratory. Haplotype estimation [Internet]. Kyoto: HLA Laboratory; 2022 [cited 2022 Jan 21]. Available from: http://hla.or.jp/med/frequency_search/ja/haplo/.

18. Kobayashi T, Iwama S, Sugiyama D, Yasuda Y, Okuji T, Ito $\mathrm{M}$, et al. Anti-pituitary antibodies and susceptible human leukocyte antigen alleles as predictive biomarkers for pituitary dysfunction induced by immune checkpoint inhibitors. J Immunother Cancer 2021;9:e002493.

19. Ishi A, Tanaka I, Iwama S, Sakakibara T, Mastui T, Kobayashi $\mathrm{T}$, et al. Efficacies of programmed cell death 1 ligand 1 blockade in non-small cell lung cancer patients with acquired resistance to prior programmed cell death 1 inhibitor and development of diabetic ketoacidosis caused by two different etiologies: a retrospective case series. Endocr J 2021;68:613-20.

20. Stamatouli AM, Quandt Z, Perdigoto AL, Clark PL, Kluger $\mathrm{H}$, Weiss SA, et al. Collateral damage: insulin-dependent diabetes induced with checkpoint inhibitors. Diabetes 2018; 67:1471-80.
21. Hernando-Calvo A, Garcia-Alvarez A, Villacampa G, Ortiz C, Bodet D, Garcia-Patos V, et al. Dynamics of clinical biomarkers as predictors of immunotherapy benefit in metastatic melanoma patients. Clin Transl Oncol 2021;23:311-7.

22. Jiang M, Peng W, Pu X, Chen B, Li J, Xu F, et al. Peripheral blood biomarkers associated with outcome in non-small cell lung cancer patients treated with nivolumab and durvalumab monotherapy. Front Oncol 2020;10:913.

23. Mertoglu C, Gunay M. Neutrophil-Lymphocyte ratio and platelet-lymphocyte ratio as useful predictive markers of prediabetes and diabetes mellitus. Diabetes Metab Syndr 2017;11 Suppl 1:S127-31.

24. Erlich H, Valdes AM, Noble J, Carlson JA, Varney M, Concannon P, et al. HLA DR-DQ haplotypes and genotypes and type 1 diabetes risk: analysis of the type 1 diabetes genetics consortium families. Diabetes 2008;57:1084-92.

25. Kawabata Y, Ikegami H. Genetics of fulminant type 1 diabetes. Diabetol Int 2020;11:315-22.

26. Miyoshi Y, Ogawa O, Oyama Y. Nivolumab, an anti-programmed cell death-1 antibody, induces fulminant type 1 diabetes. Tohoku J Exp Med 2016;239:155-8.

27. Okamoto M, Okamoto M, Gotoh K, Masaki T, Ozeki Y, Ando H, et al. Fulminant type 1 diabetes mellitus with antiprogrammed cell death-1 therapy. J Diabetes Investig 2016; 7:915-8.

28. Usui $Y$, Udagawa $H$, Matsumoto S, Imai K, Ohashi K, Ishibashi M, et al. Association of serum anti-GAD antibody and HLA haplotypes with type 1 diabetes mellitus triggered by nivolumab in patients with non-small cell lung cancer. J Thorac Oncol 2017;12:e41-3.

29. Ishikawa K, Shono-Saito T, Yamate T, Kai Y, Sakai T, Shimizu F, et al. A case of fulminant type 1 diabetes mellitus, with a precipitous decrease in pancreatic volume, induced by nivolumab for malignant melanoma: analysis of HLA and CTLA-4 polymorphisms. Eur J Dermatol 2017;27:184-5.

30. Tsang V, McGrath RT, Clifton-Bligh RJ, Scolyer RA, Jakrot V, Guminski AD, et al. Checkpoint inhibitor-associated autoimmune diabetes is distinct from type 1 diabetes. J Clin Endocrinol Metab 2019;104:5499-506.

31. de Filette J, Pen JJ, Decoster L, Vissers T, Bravenboer B, Van der Auwera BJ, et al. Immune checkpoint inhibitors and type 1 diabetes mellitus: a case report and systematic review. Eur J Endocrinol 2019;181:363-74.

32. Nishimaki K, Kawamura T, Inada H, Yagawa K, Nose Y, Nabeya N, et al. HLA DPB $1 * 0201$ gene confers disease susceptibility in Japanese with childhood onset type I diabe- 
tes, independent of HLA-DR and DQ genotypes. Diabetes Res Clin Pract 2000;47:49-55.

33. Varney MD, Valdes AM, Carlson JA, Noble JA, Tait BD, Bonella P, et al. HLA DPA1, DPB1 alleles and haplotypes contribute to the risk associated with type 1 diabetes: analysis of the type 1 diabetes genetics consortium families. Diabetes 2010;59:2055-62.

34. Inaba H, De Groot LJ, Akamizu T. Thyrotropin receptor epitope and human leukocyte antigen in Graves' disease. Front Endocrinol (Lausanne) 2016;7:120.

35. Inaba H, Martin W, Ardito M, De Groot AS, De Groot LJ. The role of glutamic or aspartic acid in position four of the epitope binding motif and thyrotropin receptor-extracellular domain epitope selection in Graves' disease. J Clin Endocrinol Metab 2010;95:2909-16.

36. Yoneda S, Imagawa A, Hosokawa Y, Baden MY, Kimura T,
Uno S, et al. T-lymphocyte infiltration to islets in the pancreas of a patient who developed type 1 diabetes after administration of immune checkpoint inhibitors. Diabetes Care 2019;42:e116-8.

37. Lu J, Liu J, Li L, Lan Y, Liang Y. Cytokines in type 1 diabetes: mechanisms of action and immunotherapeutic targets. Clin Transl Immunology 2020;9:e1122.

38. Purohit S, Sharma A, Hopkins D, Steed L, Bode B, Anderson SW, et al. Large-scale discovery and validation studies demonstrate significant reductions in circulating levels of IL8, IL-1Ra, MCP-1, and MIP-1 $\beta$ in patients with type 1 diabetes. J Clin Endocrinol Metab 2015;100:E1179-87.

39. Hong AR, Yoon JH, Kim HK, Kang HC. Immune checkpoint inhibitor-induced diabetic ketoacidosis: a report of four cases and literature review. Front Endocrinol (Lausanne) 2020;11:14 . 